\title{
Occlusion of the vertebral artery
}

\author{
TETSUO TATSUMI AND HENRY A. SHENKIN
}

From Department of Neurosurgery, Episcopal Hospital, Philadelphia, Pa.

The angiographic demonstration of an occluded vertebral artery in a patient with a brain-stem syndrome was first reported by Riechert in 1952, and similar cases have been described by various authors subsequently. The common site of the vertebral occlusion was in the area between the arch of the atlas and the junction of the vertebral arteries. Since routine brachial angiography has been initiated in this clinic, occlusion of the vertebral artery at this site has been noticed more frequently than expected. Despite good visualization of the vertebral artery, the injected contrast material sometimes stopped before the junction of the vertebral arteries, and the basilar artery was not demonstrated in a number of patients who did not have clinical evidence of vertebral or basilar artery occlusion. This observation has been mentioned by Sutton and Hoare (1951), and by Krayenbühl and Yaşargil (1957a) and was considered by them to be due to technical failure or vascular spasm. This explanation did not seem satisfactory for many instances in which we encountered an apparent vertebral artery occlusion. Therefore a series of 150 consecutive brachial angiograms has been reviewed in order to determine the frequency with which occlusion of the vertebral artery with or without symptoms occurs, and if possible to elucidate further its clinical and physiological significance.

\section{METHOD}

The initial 100 brachial angiograms were carried out by a percutaneous catheterization technique (Shenkin, Tatsumi, and Bantley, 1962) and the subsequent 50 using a direct percutaneous method as suggested by Siqueira, Karras, Cannon, and Bucy (1962). The latter method was not only less time consuming but seldom if ever interfered with the quality of the brachial pulse after the study was completed. A thin-wall 18 gauge needle was introduced into the brachial artery percutaneously at the antecubital fossa and connected to an automatic injector. Fifty-five millilitres of $60 \%$ methylglucamine diatrizoate (Renografin) were injected into the brachial artery with a pressure of $425 \mathrm{lb}$. The first film was exposed when $30 \mathrm{ml}$. of the dye had been injected and a series of six films was taken at one-second intervals in the antero-posterior and lateral projections simultaneously. The right brachial injection

${ }^{1}$ This investigation was supported in part by Public Health Service research grant HE-06753 from the National Heart Institute. usually demonstrated the right carotid and vertebral systems, and the left brachial injection demonstrated only the left vertebral system.

One hundred and fifty consecutive patients were studied with the above methods and the series is composed of 27 patients suspected of harbouring a brain tumour, 30 patients with a space-occupying lesion, 32 cases of subarachnoid haemorrhage, and 61 patients with cerebrovascular disease. Of these 150 cases, 15 patients were subjected to bilateral brachial angiography for the following reasons:

1 Six patients suffering from subarachnoid haemorrhage failed to demonstrate the basilar artery by right brachial angiography.

2 Seven patients representing a suspected vascular lesion within the brain-stem failed to demonstrate the basilar artery by a unilateral brachial injection.

3 Studies in two patients had already demonstrated multiple occlusive disease of major cerebral vessels and they were thought deserving of complete evaluation.

\section{RESULTS}

In this series of 150 cases, 37 patients ( $24 \%$ ) had an apparent occlusion of one vertebral artery at the level of the foramen magnum. Of these 37 cases, in 19 the basilar artery did not fill at all and in 18 patients the basilar artery was only faintly visualized, seen only in the lateral view. Fifteen of these 37 patients were examined by an opposite brachial artery injection for the reasons mentioned above. In 12 of these 15 cases, excellent visualization of the opposite vertebral and basilar arteries and their branches was obtained.

Of these 12 patients, seven (nos. 1-7, Table I) had no associated brain-stem symptoms or signs despite the apparent unilateral vertebral artery occlusion in the angiogram. The vertebral arteries of these patients ended smoothly at the level of the foramen magnum (Fig. 1a). The other five patients presented various brain-stem syndromes: two (nos. 8 and 9, Table I) had a corresponding dorsolateral medullary syndrome, two others (nos. 10 and 11, Table I) exhibited a paramedian ponto-medullary syndrome on the occluded side, and one patient (no. 12, Table I) had vestibulo-cerebellar symptoms. In two of these five patients, the vertebral artery ended irregularly and can be described as having a mouse-bitten 
appearance (Fig. 2). In two others the vertebral artery ended abruptly at the origin of the posterior inferior cerebellar artery and the latter appeared to be a continuation of the vertebral artery (Fig. 3a). In the remaining patient of this group, the vertebral artery, which was very small, ended smoothly.

In three patients (Table I, nos. 13,14, and 15) the basilar artery was not filled by bilateral brachial injection and they presented a syndrome of progressive bulbar palsy. They were considered to have bilateral vertebral or basilar artery occlusions. In two (nos. 13 and 14) one of the vertebral arteries was not visualized at all and the other vertebral artery ended smoothly at the mid or upper cervical level. In one patient (no. 15) a good vertebral artery on both sides was shown but both ended at their junction. A retrograde filling of the basilar artery through the posterior communicating artery was noticed in one of these three patients (no. 13, Fig. 4). A collateral circulation from the posterior inferior cerebellar artery to the superior cerebellar artery was noticed in another patient (no. 15, Fig. 6).

The right and left vertebral arteries were compared in those 15 patients who were subjected to bilateral brachial angiography. This comparison showed that the side of the occluded vertebral artery was always smaller than the patent one. The sizes of the vertebral arteries in these 15 patients were measured at the mid-cervical level and are recorded in Table I.

\section{TABLE I}

SIZE OF VERTEBRAL ARTERIES MEASURED AT THE MIDCERVICAL LEVEL AFTER BILATERAL BRACHIAL ANGIOGRAPHY AND CORRESPONDING CLINICAL SYNDROMES IN PATIENTS WITH OCCLUSION OR 'APPARENT' OCCLUSIONS OF A VERTEBRAL ARTERY.

\begin{tabular}{rccl} 
Case No. & $\begin{array}{r}\text { Right } \\
(\mathrm{mm} .)\end{array}$ & $\begin{array}{l}\text { Left } \\
(\mathrm{mm} .)\end{array}$ & $\begin{array}{l}\text { Corresponding } \\
\text { Clinical Syndrome }\end{array}$ \\
\hline 1 & $3^{1}$ & 6 & None \\
2 & 7 & 4 & None \\
3 & 4 & 6 & None \\
4 & 2 & 5 & None \\
5 & 3 & 5 & None \\
6 & 2 & 5 & None \\
7 & 2 & $4 \cdot 5$ & None \\
8 & 6 & 2 & Dorsolateral medullary \\
9 & 2 & 5 & Dorsolateral medullary \\
10 & 5 & 2 & Paramedian ponto-medullary \\
11 & 2 & 4 & Paramedian ponto-medullary \\
12 & $1 \cdot 5$ & 5 & Vestibulo-cerebellar \\
13 & 0 & 2 & Bulbar palsy \\
14 & 2 & 0 & Bulbar palsy \\
15 & 3 & 5 & Bulbar palsy
\end{tabular}

${ }^{1}$ Italics indicate the side of the occlusion.

In summary, in this series of 150 consecutive cases in which the vertebral arteries were visualized there were 37 instances of occlusion $(24 \%), 29$ of these patients $(19 \%)$ having no symptoms, five $(3 \%)$ with brain-stem symptoms, and three $(2 \%)$ with basilar $\frac{\text { O }}{Z}$ artery occlusion and symptoms thereof.

\section{ILLUSTRATIVE CASES}

CASE 1 A 36-year-old woman suddenly developed severe headache 10 days before admission to the hospital. She gradually improved but about a week after the onset she $\frac{\rho}{\vec{S}}$ again developed severe headache which was followed by a transient loss of consciousness and mental confusion. Examination revealed her to be neurologically normal

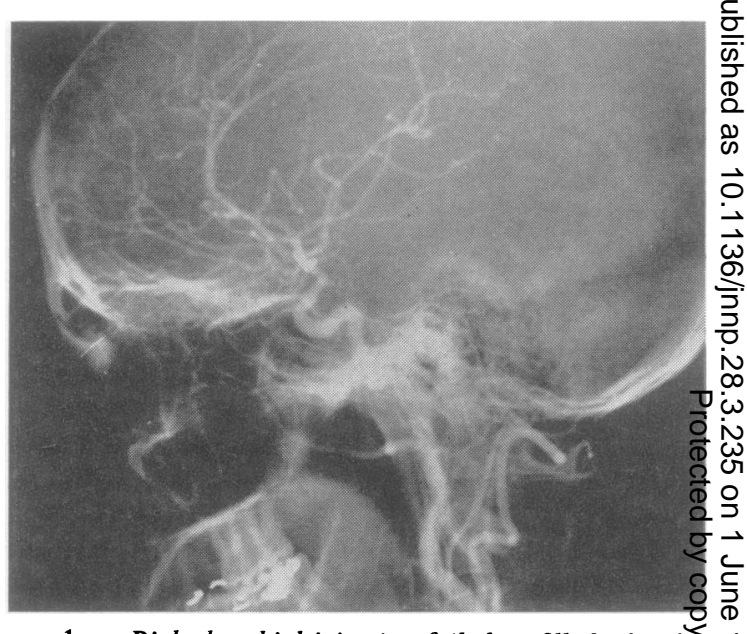

FIG. 1a. Right brachial injection failed to fill the basilax. $\overrightarrow{0}$ artery. The right vertebral artery ends smoothly at the level of the foramen magnum.

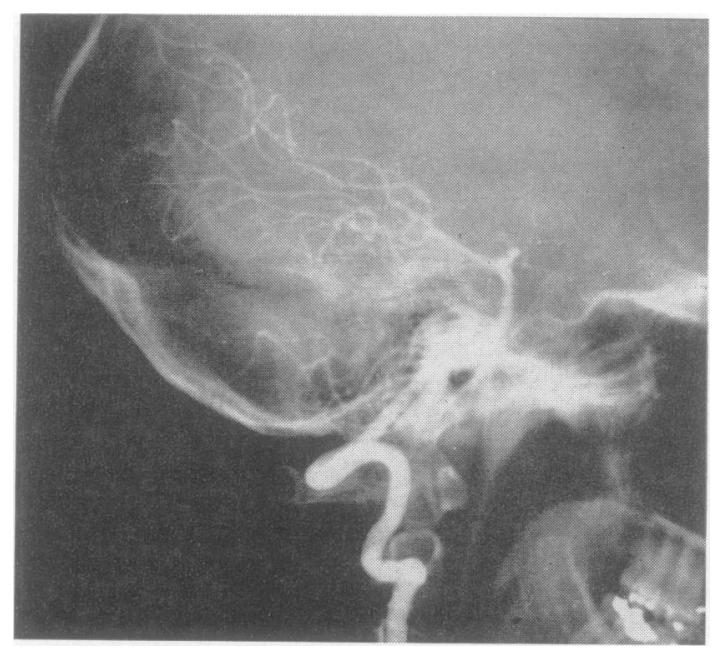

FIG. 1b. Left brachial injection filled the entire vertebral system. Notice the left vertebral artery is larger than the right in Fig. Ia (same patient as Fig. la). 
except for marked nuchal rigidity. Her blood pressure was $130 / 90 \mathrm{~mm} . \mathrm{Hg}$ and a lumbar puncture revealed bloody spinal fluid with an initial pressure of $290 \mathrm{~mm}$. of water. Right brachial angiography demonstrated an aneurysm on the right internal carotid artery at the origin of the posterior communicating artery. The right vertebral artery was well visualized but ended smoothly at the level of the foramen magnum (Fig. 1a). The size of the right vertebral artery was $3 \mathrm{~mm}$. in diameter at the midcervical level. Left brachial angiography demonstrated an excellent filling of the left vertebral and the basilar artery and their branches (Fig. 1b). The size of the left vertebral artery was $6 \mathrm{~mm}$. in diameter.

CASE 8 A 49-year-old man suddenly developed vertigo, nausea, and vomiting on the day before admission to the hospital. On the morning of admission the vertigo increased and he lost consciousness for a few minutes and thereafter he was ataxic. Neurological examination disclosed a left Horner's syndrome, hypalgesia of the left side of his face and the right side of his body and extremities. Coordination of his left extremities was impaired. A lumbar puncture revealed a clear spinal fluid with an initial pressure of $180 \mathrm{~mm}$. of water. Left brachial angiography visualized a small left vertebral artery measuring $2 \mathrm{~mm}$. in diameter ending irregularly at the level of the foramen magnum (Fig. 2). Right brachial angiography demonstrated normal right vertebral and basilar arteries and their branches. The size of the right vertebral artery was $6 \mathrm{~mm}$. in diameter.

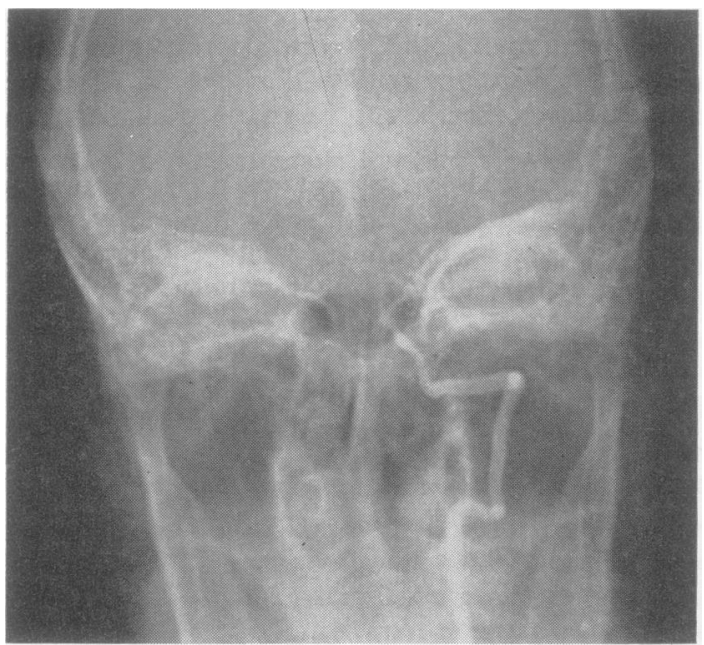

FIG. 2. Pathological occlusion of the left vertebral artery. Irregularity of the artery is seen proximal to the occluded site.

CASE 10 A 43-year-old woman suddenly became vertiginous, associated with numbness of the left side of her face about four weeks before admission to the hospital. She also complained of diplopia, diminished hearing, and staggering gait. Neurological examination

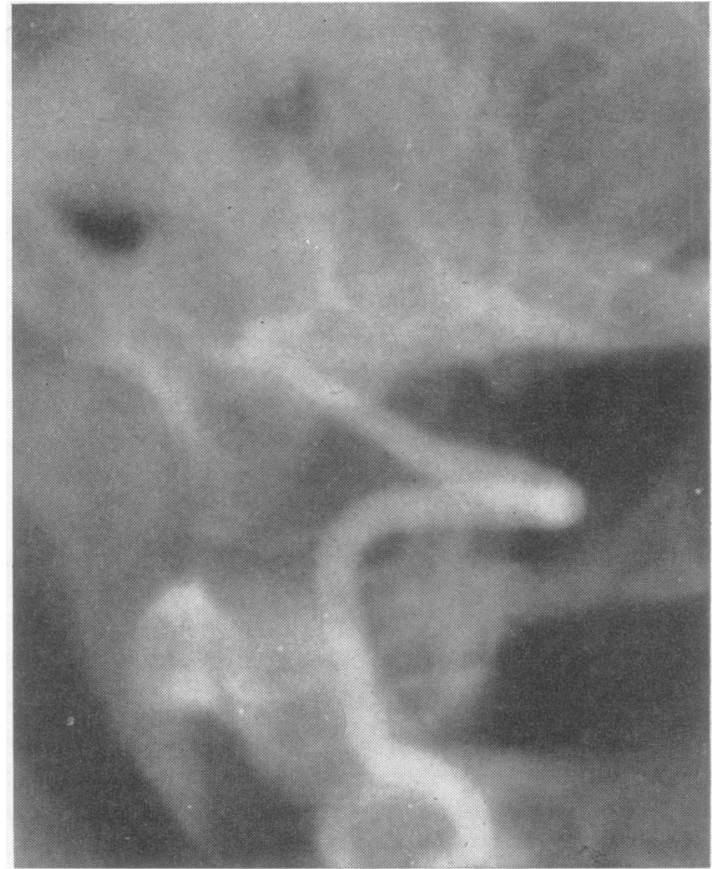

FIG. 3a. Left vertebral artery (case 10, Table I) occluded at the origin of the left posterior inferior cerebellar artery. The posterior inferior cerebellar artery appears to be $a$ continuation of the left vertebral artery.

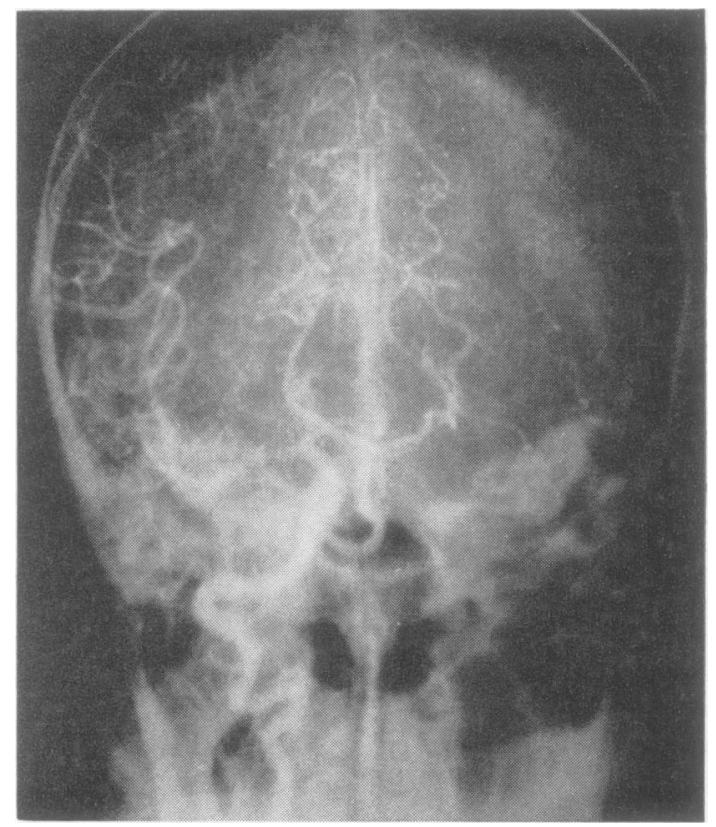

FIG. 3b. Right brachial injection in case 10 demonstrates the normal appearance of the right vertebral and basilar arteries and their branches. 
revealed a left peripheral facial weakness, left abducens weakness, and horizontal nystagmus. There was hypalgesia of the left side of her face and the right side of her body. The corneal reflex was absent on the left. The left palate was weak and her voice was hoarse. Hearing in the left ear was impaired. Coordination of her left extremities was impaired and Romberg's sign was positive. Lumbar puncture disclosed clear spinal fluid with an initial pressure of $120 \mathrm{~mm}$. of water and containing $65 \mathrm{mg}$. of protein per $100 \mathrm{ml}$. Left brachial angiography revealed a small left vertebral artery, $2 \mathrm{~mm}$. in diameter, and it ended just distal to the origin of the posterior inferior cerebellar artery. Therefore the left posterior inferior cerebellar artery appeared to be a continuation of the left vertebral artery (Fig. 3a). Right brachial angiography provided an excellent demonstration of the right vertebral and basilar arteries (Fig. 3b). The right vertebral artery measured $5 \mathrm{~mm}$. in diameter.

CASE 12 A 54-year-old man suddenly developed vertigo, headache, and vomiting followed by a marked ataxia two days before admission to the hospital. On examination he was slightly disorientated and somewhat confused and his speech was markedly dysarthric. Bilateral horizontal nystagmus was present and the neck was moderately rigid. His extremities were markedly asynergic and he was unable to walk because of ataxia. His spinal fluid was slightly bloody with an initial pressure of $180 \mathrm{~mm}$. of water. Right brachial angiography revealed a small right vertebral artery measuring $1.5 \mathrm{~mm}$. in diameter which ended smoothly at the level of the foramen magnum. Left brachial angiography demonstrated excellent filling of the left vertebral and basilar artery and the left vertebral artery measured $5 \mathrm{~mm}$. in diameter. Complete cerebral angiography gave no evidence of an intracranial aneurysm or haematoma. The patient recovered progressively but mild ataxia persisted.

CASE 13 A 55-year-old man, who had had diabetes mellitus and hypertension for 10 years, was admitted to the hospital with the chief complaint of pain in the right

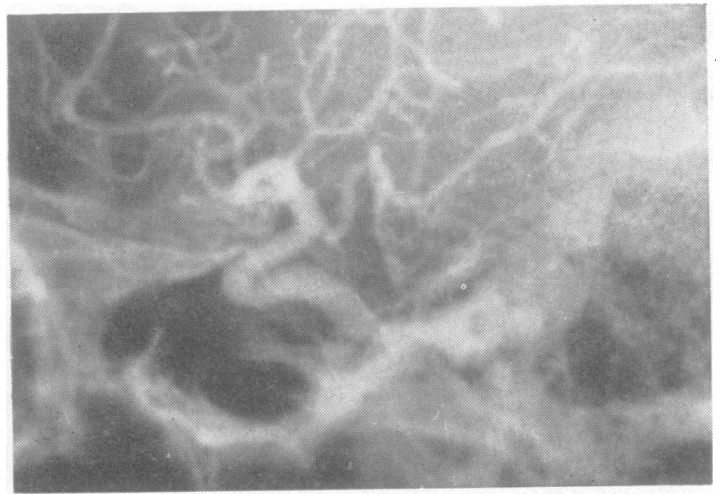

FIG. 4. Retrograde filling of the basilar artery through the posterior communicating artery in case 13 (Table I), with vertebral arteries bilaterally occluded. lower extremity. On the day after admission he developed slurred speech, difficulty with swallowing, and weakness of both lower extremities. Neurological examination revealed his speech to be dysarthric and cerebellar coordination to be impaired bilaterally. Both lower extremities were extremely weak and his knee and ankle reflexes were absent bilaterally. Right brachial angiography did not show the right vertebral artery at all. Left brachial angiography showed a small left vertebral artery measuring $2 \mathrm{~mm}$. in diameter, ending abruptly at the mid-cervical level. However, a retrograde filling of the basilar artery through the posterior communicating artery was demonstrated (Fig. 4). This patient had to be fed by a nasal feeding tube for several months but thereafter made a reasonably complete recovery.

\section{DISCUSSION}

Technical factors in the performance of vertebral angiography, such as hyperextension of the neck or improperly positioning the needle causing spasm of the artery, has been offered as the basis for the appearance of an apparent occlusion of this vessel. However, these factors were eliminated in our series by the use of brachial injections and care never to hyperextend the neck during the procedure; nevertheless, vertebral occlusions were still encounteredo relatively frequently. Sutton and Hoare (1951) sug $\mathbb{D}$ G gested that a higher pressure in the opposite vertebral次 응 artery than in the injected side could explain some in- $\overrightarrow{2}$ stances of an apparent vertebral artery occlusion.

Examination of both vertebral arteries in instances of unilateral vertebral occlusion has revealed that the obstructed vertebral artery was always smaller than its opposite (Table I). Because asymmetry of the vertebral arteries is so frequent and because it occurs also in young adults apparently free of vascular disease, it can be presumed to be an anatomical variation rather than a result of pathological change. Further, an anatomical study by Adachi (1928) indicated that the vertebral arteries were equal in size in only $28.6 \%$ of 182 cases; the right was the larger in $25.8 \%$ and the left larger in $45.8 \%$ of cases. From this evidence, it may be concluded that inequality of the vertebral arteries in most individuals is a normal anatomical state and this inequality may serve as the basis for the frequency of an 'apparent occlusion' of a vertebral artery. The velocity of the blood flow should be slower in the larger vessel, and, according to Bernouilli's principle, the lateral pressure of the blood in this vessel would then be proportionately greater than in the smaller vessel. This increased lateral pressure in the larger vertebral artery could block the flow from the smaller vertebral artery at their junction. If the difference in size between the two vertebral arteries is not too great, the smaller flow may get into the basilar artery against the larger flow, and result in a small streak as 


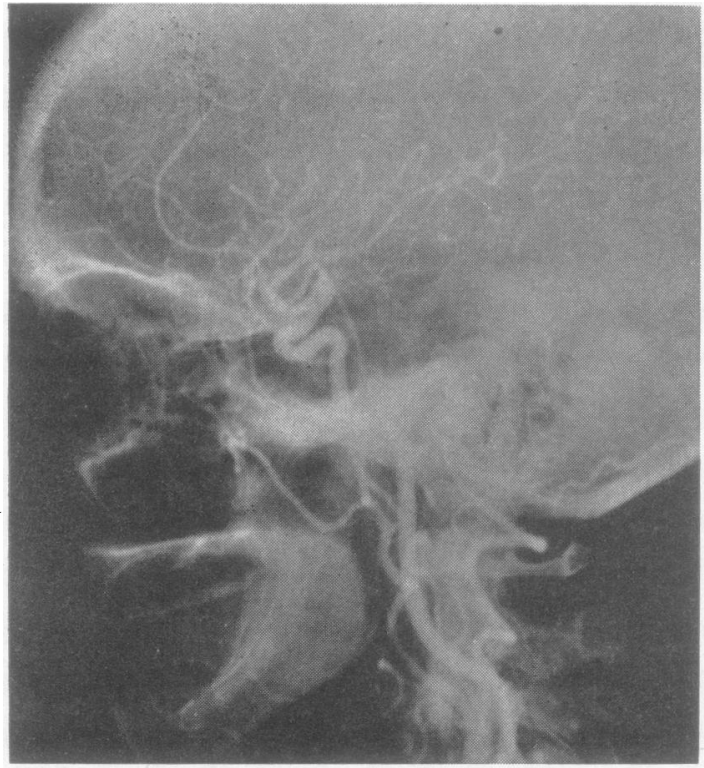

FIG. 5a. Faint filling of the basilar artery following right brachial injection.

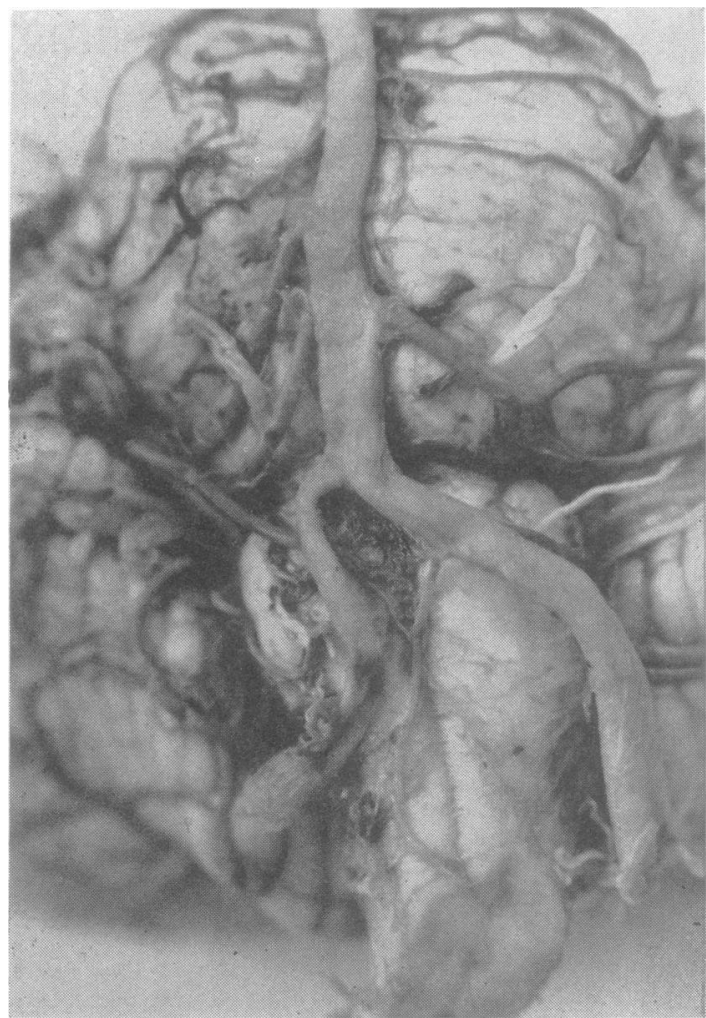

FIG. 5b. Necropsy of the same patient as in Fig. $5 a$ reveals the right vertebral artery to be smaller than the left. a laminar flow (McDonald, 1960) along the basilar artery wall on the side of the smaller vertebral vessel. This would then be visualized as a thin basilar artery in the brachial angiogram done from this side (Figs. $5 a$ and $5 b$ ).

It is important to differentiate a physiological occlusive phenomenon from pathological occlusion of a vertebral artery and the following four points might be helpful:-

1 In instances of physiological occlusion the basilar artery often can be seen faintly, especially on the lateral projection (Fig. 5a).

2 A pathologically occluded vessel frequently has an irregular or 'mouse-bitten' termination (Fig. 2). When the occlusion is physiological, the vessel most often terminates smoothly (Fig. 1a).

3 The presence of a collateral circulation filling the basilar artery and its branches strongly indicates a pathological occlusion of the vertebral circulation. Retrograde filling of the basilar artery through the posterior communicating arteries was noted in one instance of bilateral vertebral artery occlusion (Fig. 4) and this has been reported previously by Van Der Zwan (1954). All do not agree that this occurs only when there is basilar or vertebral artery obstruction. A collateral circulation from the posterior inferior cerebellar artery to the superior cerebellar artery was demonstrated in one instance of basilar artery occlusion (Fig. 6). Dilatation of the muscular branches from the external carotid artery and its communication to the vertebral artery was mentioned by Krayenbühl and Yaşargil (1957b).

4 When the vertebral artery is occluded just distal to the origin of the posterior inferior cerebellar

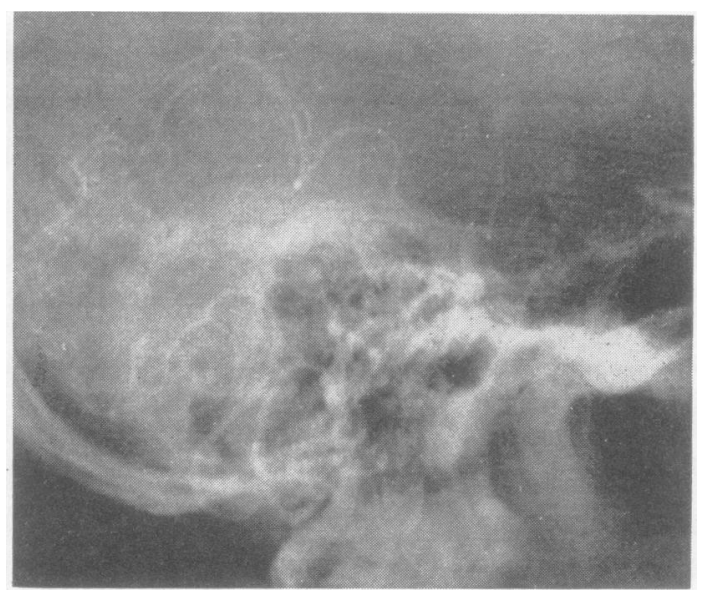

FIG. 6. Collateral circulation from the posterior inferior cerebellar artery to the superior cerebellar artery in a patient with occlusion of the vertebral arteries bilaterally (case 15). 
artery, the posterior inferior cerebellar artery will appear to be a continuation of the vertebral artery (Fig. 3a).

The presence of clinical symptoms indicates that an observed occlusion of a vertebral artery is of the pathological type. The absence of clinical symptoms does not necessarily mean that the block of a vertebral artery is physiological. It is always possible that principal branches are spared and fill from either proximal or distal or collateral channels not always visualized in the arteriographic study. A variety of brain-stem syndromes has been reported to accompany unilateral vertebral artery occlusion. Krayenbühl and Yaşargil (1957b) have demonstrated occlusion of the vertebral artery in 12 patients with typical dorso-lateral medullary syndromes and in three patients with medullo-pontine-cerebellar symptoms. Fisher, Karnes, and Kubik (1961) studied 16 cases of Wallenberg's syndrome at necropsy and found that the vertebral artery was occluded just proximal to the vertebral-basilar junction in 12 cases and occlusion of the posterior inferior cerebellar artery alone was found in only two cases. They concluded, therefore, that occlusion of the vertebral artery rather than of the posterior inferior cerebellar artery is most often responsible for this condition. Currier, Schneider, and Preston (1962) also have recently reported seven cases of Wallenberg's syndrome with angiographic evidence of an occluded vertebral artery. From these reports and our own experiences it appears clear that the dorso-lateral medullary syndrome results principally from unilateral occlusion of a vertebral artery rather than from posterior inferior cerebellar artery occlusion alone. Unilateral vertebral artery occlusion also not infrequently can be responsible for medullo-pontine or cerebellar symptoms. The variations in the symptoms, as noted by Krayenbühl and Yaşargil (1957b), are probably the result of the status of the collateral circulation in each individual, or possibly due to anatomical variations of the vertebral artery or its branches.

The three patients in whom the basilar artery did not fill from either vertebral artery, the conditions were associated with progressive bulbar palsy. Two of these patients died and proved to have a thrombosis of the basilar artery at necropsy. One recovered apparently by the retrograde filling of the basilar artery through the posterior communicating artery. From our present study it is obvious that one should not make a diagnosis of basilar artery insufficiency or thrombosis based on one-sided vertebral angiography only.

\section{SUMMARY}

The appearance of the vertebral and basilar arteries was reviewed in a series of 150 consecutive cases of brachial angiography. In $37(24 \%)$ patients the basilar artery was not visualized despite good visualization of the vertebral artery. Twenty-nine (19\%) patients had an occlusion of a unilateral vertebral artery unassociated with corresponding neurological symptoms or signs and the obstructed vertebral artery ended smoothly. Five patients $(3 \%)$ were associated with brain-stem syndromes and their vertebral arteries terminated irregularly or appeared to be continuous with the posterior inferior cerebellar artery. Three patients $(2 \%)$ were found to have bilateral vertebral or basilar artery occlusions.

In some instances it is suggested that an occlusion of a unilateral vertebral artery visualized arteriographically can be due to a haemodynamic phenomenon based on the fact that the occluded vertebral artery is smaller than its opposite and apparently patent vessel.

\section{REFERENCES}

Adachi, B. (1928). Das Arteriensystem der Japainer, vol. I. Maruzen Kyoto and Tokyo.

Currier, R. D., Schneider, R. C., and Preston, R. E. (1962). Angio graphic findings in Wallenberg's lateral medullary syndrome? J. Neurosurg., 19, 1058-1067.

Fisher, C. M., Karnes, W. E., and Kubik, C. S. (1961). Latera medullary infarction-the pattern of vascular occlusion. J. Neuropath. exp. Neurol., 20, 323-379.

Krayenbühl, H., and Yaşargil, M. G. (1957a). Die vaskulären Erkrankungen im Gebiet der Arteria vertebralis und Arteria basialis. Thieme, Stuttgart.

_ graphischen Bild. Dtsch. Z. Nervenheilk., 177, 103-116.

McDonald, D. A. (1960). Blood Flow in Arteries. Williams \& Wilkins, Baltimore.

Riechert, T. (1952). Über arteriographisch nachgewiesene Verschlüsse der Arteria vertebralis. Arch. Psychiat. Nervenkr., 188, 126-130.

Shenkin, H. A., Tatsumi, T., and Bantley, D. (1962). Simplified method for total cerebral angiography. J. Amer. med. Ass. 182, 132-135.

Siqueira, E. B., Karras, B. G., Cannon, A. H., and Bucy, P. C. (1962). Percutaneous brachial cerebral angiography. J. Neurosurg., 19, 1050-1057.

Sutton, D., and Hoare, R. D. (1951). Percutaneous vertebral arteriography. Brit. J. Radiol., 24, 589-597.

Van Der Zwan, A. (1954). Angiographic diagnosis of vertebral artery thrombosis. J. Neurol. Neurosurg. Psychiat., 17, 189-190. 